\title{
New Technique of Wireless Sensor Networks Localization based on Energy Consumption
}

\author{
Anouar Abdelhakim Boudhir \\ Laboratory LIST, ERIT \\ FST of Tangier, Morocco
}

\author{
Bouhorma Mohamed \\ Laboratory LIST,ERIT \\ FST of Tangier, Morocco
}

\author{
Ben Ahmed Mohamed \\ Laboratory LIST,ERIT \\ FST of Tangier, Morocco
}

\begin{abstract}
Localization has newly received research interest due to the success of the emerging wireless sensor network (WSN) technology. This interest is expected to grow further with the proliferation of wireless sensor network applications such as medicine, military, transport... In this context routing, protocols and technologies of communication on those wireless area are enormously applied to sensor networks in order to improve the quality of service and communication. Related to this work, we present on this article a briefly some techniques of localization techniques on wireless sensor networks and propose a localization method based on the angle of transmission of directional antenna and the energy consumption of the node when sending a data. Then we compare the mentioned method to TDOA technique (Time Differential Of Arrival) applied on the context of mobility characterizing the transport domain and compare their performance when localizing sensors in this kind of applications.
\end{abstract}

\section{General Terms}

Energy consumption, WSN, Localization techniques, TDOA, AOA, RSS, NS2 ...

\section{Keywords}

Localization, WSN, ZigBee, NS2

\section{INTRODUCTION}

A Wireless Sensor Network (WSN) is formed by hundreds of small, low-cost nodes which have limitations in memory, energy, and processing capacity. In this type of networks, one of the main problems is to locate each node. Recent advances in wireless communications and electronics have enabled the development of low-cost, low-power and multi-functional sensors that are small in size and communicate in short distances. Cheap, smart sensors, networked through wireless links and deployed in large numbers, provide unprecedented opportunities for monitoring and controlling homes, cities, and the environment. In addition, networked sensors have a broad spectrum of applications in the defense area, generating new capabilities for reconnaissance and surveillance as well as other tactical applications. Self-localization capability is a highly desirable characteristic of wireless sensor networks. In environmental monitoring applications such as bush fire surveillance, water quality monitoring and precision agriculture, the measurement data are meaningless without knowing the location from where the data are obtained. Moreover, location estimation may enable a myriad of applications such as inventory management, transport, intrusion detection, road traffic monitoring, health monitoring, reconnaissance and surveillance.

\section{LOCALIZATION AND MEASUREMENT TECHNIQUES}

\subsection{Localization Techniques}

Advances in micro-electro-mechanical systems have triggered an enormous interest in wireless sensor networks (WSN). WSN are formed by large numbers of densely deployed nodes enabled with sensing and actuating capabilities. These nodes have very limited processing and memory capabilities, limited energy resources and it is envisioned that they will be mass produced, to reduce costs. Several challenging problems exist in wireless sensor networks. Among these is how to obtain location information for sensor nodes and events present in the network. From this perspective, we categorize the localization problem as: node localization, target localization and location service. Node localization is the process of determining the coordinates of the sensor nodes in the WSN. Target localization is the process of obtaining the coordinates of an event or a target present in the sensor network. The location of a target can be obtained either passively (the nodes sense the target) or actively, when the target cooperates and communicates with the sensor network.Node localization is a complicated and important problem for wireless sensor networks (WSN). The aspects of this problem that have challenged the research community can be summarized as follows:

Assumptions - The node localization problem remains a difficult challenge to be solved practically. To make the problem practically tractable, its complexity had to be reduced, by making simplifying assumptions. As a result, many localization schemes proposed solutions that are based on assumptions that do not always hold or are not practical. Examples of such assumptions are: circular radio range, symmetric radio connectivity, additional hardware (e.g., ultrasonic), lack of obstructions, lack of line-of-sight, no multipath and flat terrain. Localization Protocol Design - The problem of localization in WSN is further complicated by the large number of parameters that need to be considered when designing a localization system for a particular WSN deployment. Among these parameters are: the deployment method for the sensor network; the existence of a line-of-sight between sensor nodes and a remote, central point; the time required by the localization scheme; the presence of reference points (anchors) in the network, and the density; the cost for localization, represented by additional hardware (form factor) and energy expenditure (messages exchanged or time necessary for localization).

Sensor network localization algorithms estimate the locations of sensors with initially unknown location information by using knowledge of the absolute positions of a few sensors and intersensor measurements such as distance and bearing measurements. Sensors with known location information are 
called anchors and their locations can be obtained by using a global positioning system (GPS), or by installing anchors at points with known coordinates. In applications requiring a global coordinate system, these anchors will determine the location of the sensor network in the global coordinate system. In applications where a local coordinate system suffices (e.g., smart homes), these anchors define the local coordinate system to which all other sensors are referred. Because of constraints on the cost and size of sensors, energy consumption, implementation environment (e.g., GPS is not accessible in some environments) and the deployment of sensors (e.g., sensor nodes may be randomly scattered in the region), most sensors do not know their locations. These sensors with unknown location information are called non-anchor nodes and their coordinates will be estimated by the sensor network localization algorithm.

\subsection{Measurement techniques}

Measurement techniques in WSN localization can be broadly classified into three categories: AOA measurements, distance related measurements and RSS profiling techniques.

\subsubsection{Angle-of-arrival measurements}

The angle-of-arrival measurement techniques can be further divided into two subclasses: those making use of the receiver antenna's amplitude response and those making use of the receiver antenna's phase response. Beamforming is the name given to the use of anisotropy in the reception pattern of an antenna, and it is the basis of one category of AOA measurement techniques. The measurement unit can be of small size in comparison with the wavelength of the signals. One can imagine that the beam of the receiver antenna is rotated electronically or mechanically, and the direction corresponding to the maximum signal strength is taken as the direction of the transmitter. Relevant parameters are the sensitivity of the receiver and the beam width. A technical problem to be faced and overcome arises when the transmitted signal has a varying signal strength. The receiver cannot differentiate the signal strength variation due to the varying amplitude of the transmitted signal and the signal strength variation caused by the anisotropy in the reception pattern. One approach to dealing with the problem is to use a second non-rotating and omnidirectional antenna at the receiver. By normalizing the signal strength received by the rotating anisotropic antenna with respect to the signal strength received by the non-rotating omnidirectional antenna, the impact of varying signal strength can be largely removed. Fig. 1 shows an antenna array of $\mathrm{N}$ antenna elements. The adjacent antenna elements are separated by a uniform distance $d$ [1]. The distance between a transmitter far away from the antenna array and the $i^{\text {th }}$ antenna element can be approximated by:

$$
R i=R O-i d \cos \theta
$$

where R0 is the distance between the transmitter and the 0th antenna element and $\theta$ is the bearing of the transmitter with respect to the antenna array.

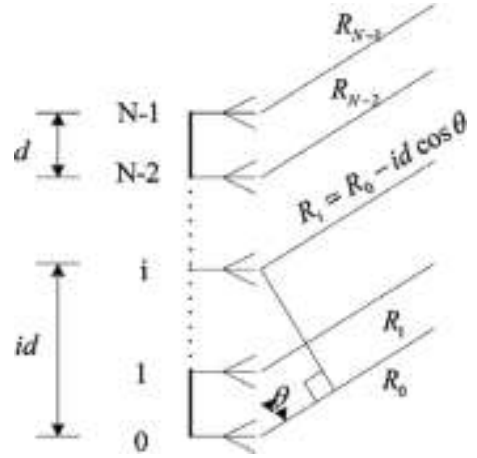

Fig. 1. An antenna array with $\mathrm{N}$ antenna elements.

\subsubsection{Distance related measurements}

Distance related measurements include propagation time based measurements, i.e., one-way propagation time measurements, roundtrip propagation time measurements and time-difference-of-arrival (TDOA) measurements. Another interesting technique measuring distance, which does not fall into the above categories, is the lighthouse approach shown in [2]. In the following paragraphs we provide further details of these techniques.

\section{- One-way propagation time and roundtrip propagation time measurements}

One-way propagation time and roundtrip propagation time measurements are also generally known as time-of-arrival measurements. Distances between neighboring sensors can be estimated from these propagation time measurements.

One-way propagation time measurements measure the difference between the sending time of a signal at the transmitter and the receiving time of the signal at the receiver. It requires the local time at the transmitter and the local time at the receiver to be accurately synchronized.

\section{- Time-difference-of-arrival measurements}

There is a category of localization algorithms utilizing TDOA measurements of the transmitter's signal at a number of receivers with known location formation to estimate the location of the transmitter. Fig. 2 shows a TDOA localization scenario with a group of four receivers at locations $r 1, r 2, r 3, r 4$ and a transmitter at rt. The TDOA between a pair of receivers $i$ and $j$ is given by:

$$
\Delta \mathrm{tij}=\mathrm{ti} \_\mathrm{tj}=\frac{1}{\mathrm{C}}\left(\left\|\mathrm{ri} \_\mathrm{rt}\right\|_{-}\left\|\mathrm{rj} \_\mathrm{rt}\right\|\right) ; \mathrm{i} \neq \mathrm{j} ;
$$

where ti and t $j$ are the time when a signal is received at receivers $i$ and $j$, respectively, $c$ is the propagation speed of the signal, and $\|$ denotes the Euclidean norm. Measuring the TDOA of a signal at two receivers at separate locations is a relatively mature field [3].

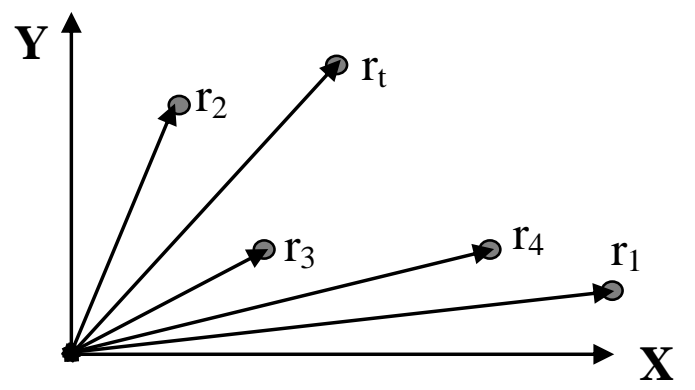

Fig. 2. Localization using time-difference-of-arrival measurements. 
Table 1. Parameters used in simulations

\begin{tabular}{|l|c|}
\hline \multicolumn{2}{|c|}{ Parameters } \\
\hline Mac/phy & $802 \_15 \_4$ \\
\hline Chanel & Wireless Channel \\
\hline Propagation & TwoRayGround \\
\hline Area & $100 * 100$ \\
\hline Traffic & FTP \\
\hline Number of nodes & 5 \\
\hline Speed of nodes & $20 \mathrm{~m} / \mathrm{s}$ \\
\hline RtPower & $0.08 \mathrm{w}$ \\
\hline TxPower & $0.4 \mathrm{w}$ \\
\hline Initial Energy & $1 \mathrm{j}$ \\
\hline
\end{tabular}

In summary, a number of measurement techniques are available for WSN localization. Which measurement technique to use for location estimation will depend on the specific application. Typically, localization algorithms based on AOA and propagation time measurements are able to achieve with acceptable accuracy than localization algorithms based on GPS measurements which consume more energy.

\subsection{Techniques comparison}

One of the most appealing problems to be solved by localization techniques is how to provide an anytime, anywhere, fine-grained, and reliable localization system to be used by transport vehicles for critical safety and emergency applications. An anytime requirement means that the localization system must be free of delays when computing the current positions of the vehicles. This requirement is critical, since the high mobility of vehicles means that slightly outdated position information cannot be used and could even be dangerous. To be available anywhere is also a challenge in localization system. It means that the localization system cannot rely only on satellite infrastructure, since it would then not work in environments without direct visibility to satellites. Also, it cannot rely only on local infrastructured localization techniques, since it would not be available in places without this infrastructure. Finally, a finegrained localization system ensures a low localization error for vehicles, which enables most critical applications to have some degree of confidence. A lot of researches focus on static sensor networks. Relatively less is known about localization in mobile sensor networks, and very few algorithms work in situations where the sensors may be static or mobile.

\section{CONTRIBUTION AND PROPOSED METHOD}

We based our work on [15] using the energy model consumption sending and receiving one byte of data from node $i$ to node $j$ over a distance $d$ meters, and we consider that the energy consumption costs:

$$
\begin{aligned}
& e_{i j}^{s}=c_{1}+c_{2} d_{i j}^{2} \\
& e_{j i}^{r}=c_{1} .
\end{aligned}
$$

We consider here that $d_{i j}$ as the variable needed to localize node destination, and by knowing the energy consumed when sending a data from node $i$ to node $j$ and using the angle of arrival $\alpha .\left(\mathrm{c}_{1}, \mathrm{c}_{2}, \mathrm{e}_{\mathrm{ij}}\right.$ and $\mathrm{e}_{\mathrm{ji}}$ are defined in [15]).

Using the equation ( 3 ) we can easily deduce de distance $d$ separate the two nodes:

$$
d_{i j}=\sqrt{\left|\frac{\mathrm{e}_{\mathrm{ij}-c_{1}}^{\mathrm{s}}}{\mathrm{c}_{2}}\right|}
$$

by calculating the distance $d$ we place the node destination $j$ on the circle where the node $i$ represent his centre. The angle of arrival serves for locating the node $j$ on the segment $S$ and reducing the probability of positioning the node on all area of the circle. Considering the segment $S$ proximately similar to line, and using the Euclidian distance $d$ between node $I$ and $j$ at the instant $t$ we denote:

$$
\begin{aligned}
d_{i j}(\mathrm{t}) & =\sqrt{\left(X_{i}-X_{j}\right)^{2}+\left(Y_{i}-Y_{j}\right)^{2}} \\
S & \cong 2 \cdot d_{i j}(t) \cdot \sin \left(\frac{\alpha}{2}\right) \\
& =2 \cdot \sqrt{\left(X_{i}-X_{j}\right)^{2}+\left(Y_{i}-Y_{j}\right)^{2}} \cdot \sin \left(\frac{\alpha}{2}\right)
\end{aligned}
$$

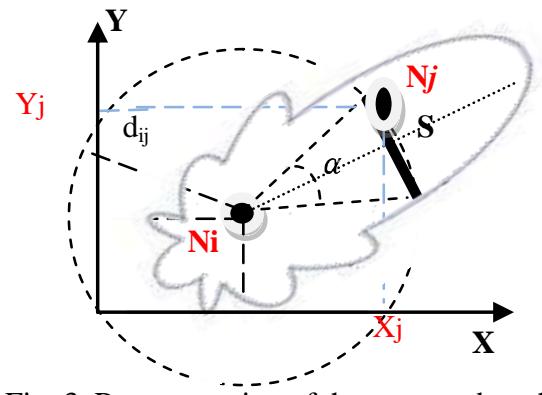

Fig. 3. Representation of the proposed method

This method (fig.3) of localization, based on two techniques (angle of directional antenna and the energy of transmission), can sufficiently locate node with reduced probability. The rate and magnitude of this probability can be neglected specially for vehicles and transport applications where the node has a considerable volume compared to the segment $\mathrm{S}$. Unlike the others techniques, the method mentioned reduce the energy consumption of nodes used aggressively in GPS techniques.

\section{SIMULATION AND RESULTS}

Using the popular tool NS2, we proceed to analyze the performance of the TDOA, and the proposed method using the standard IEEE.802.15 (ZigBee) really implemented on actual sensors using 5 mobile nodes with a speed fixed on $20 \mathrm{~m} / \mathrm{s}$ on and simulation over 100 seconds (in the mobile context).We used the model Random way Point model in the area of $100 \mathrm{~m}^{2}$ (Table.1) and the directional Antenna.

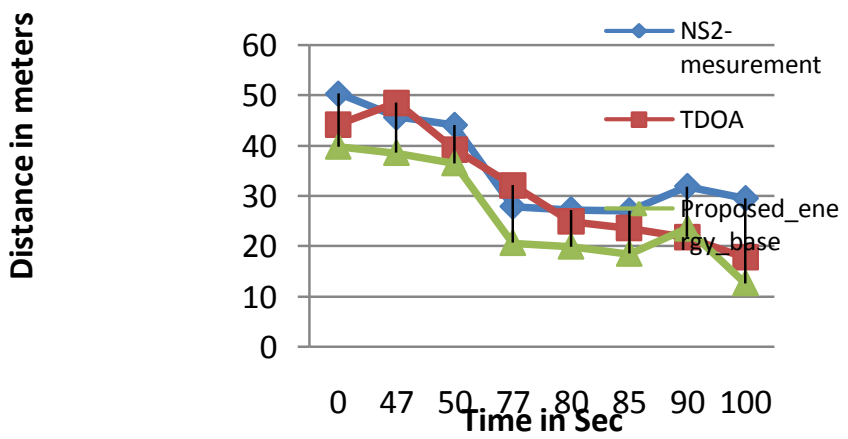

Fig. 4.Comparison of the proposed method, TDOA and NS2 measurements 


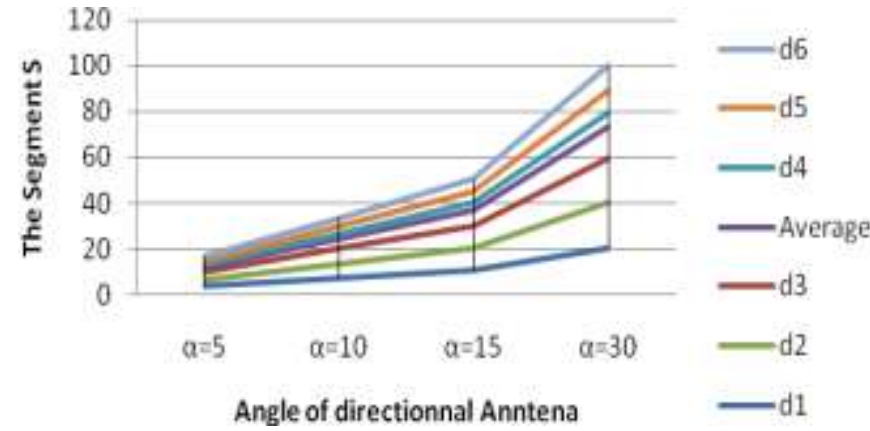

Fig. 5.Probability of positioning destination node on the segment measured by the angle $\alpha$ and $\mathrm{d} i(i=0 \rightarrow 6)$ are the distance calculated by our method

We proceed to the variation distance between node source and the four nodes destination used in the script of simulation, by function of time when nodes moves at the speed $20 \mathrm{~m} / \mathrm{s}$. The fig.4 illustrate the comparison between the proposed method, based on the energy consumed by sending 512 Kbyte of packets and calculated the distance $d$ between the nodes $i$ and $j$ using the formula based on [15], the TDOA technique based on equation (2), and the NS2-mesurement using the Euclidian distance mentioned on the equation (6). By using the distance calculated on our method, we can deduce easily the angle $\alpha$ from the equation (7) and we can see that when $\alpha$ decrease (fig.5) we obtain a small segment of $S$ and by contrast this distance increase when $\alpha$ is high. Because of this, our method gives a good results when $\alpha$ is small. Huns we reduce the segment $S$ and the probability to localize the destination in this section which can proportional to the dimensions and size of the mobile or vehicle were is implemented the sensors.

\section{CONCLUSION AND FUTURE WORK}

We presented in this paper a measurement technique for localization, applied to sensor network in transport domain which knows a high mobility. This technique is based on the energy consumption of sensor node and the angle $\alpha$ of transmission of a directional antenna, and compared to TDOA technique .We recommend, in our method, to use a small angle of the antenna in order to localize approximately and with high probability nodes implemented in applications like transport and vehicles known by considerable dimensions and size. For the future work, we can think how combining AOA, TDOA and the presented method using the UWB standards for localization under NS2.

\section{REFERENCES}

[1]. T. Rappaport, J. Reed, B. Woerner, Position location using wireless communications on highways of the future, IEEE Communications Magazine.

[2]. K. Romer, The lighthouse location system for smart dust, in: Proceedings of MobiSys 2003 (ACM/USENIX Conference on Mobile Systems, Applications, and Services), pp. 15-30.
[3]. G. Carter, Time delay estimation for passive sonar signal processing, IEEE Transactions on Acoustics, Speech, and Signal Processing 29 (3) (1981) 463-470.

[4]. F. Gustafsson, F. Gunnarsson, Mobile positioning using wireless networks: possibilities and fundamental limitations based on available wireless network measurements, IEEE Signal Processing Magazine 22 (4) (2005) 41-53.

[5]. S. Guolin, C. Jie, G. Wei, K.J.R. Liu, Signal processing techniques in network-aided positioning: a survey of stateof- the-art positioning designs, IEEE Signal Processing Magazine 22 (4) (2005) 12-23.

[6]. J.-Y. Lee, R. Scholtz, Ranging in a dense multipath environment using an UWB radio link, IEEE Journal on Selected Areas in Communications 20 (9) (2002) 16771683 .

[7]. S.V. Schell, W.A. Gardner, High-resolution direction finding, Handbook of Statistics.

[8]. R. Klukas, M. Fattouche, Line-of-sight angle of arrival estimation in the outdoor multipath environment, IEEE Transactions on Vehicular Technology 47 (1) (1998) 342 351.

[9]. T. Rappaport, J. Reed, B. Woerner, Position location using wireless communications on highways of the future, IEEE Communications Magazine 34 (10) (1996) 33-41

[10].B. Halder, M. Viberg, T. Kailath, An efficient non-iterative method for estimating the angles of arrival of known signals, in: The Twenty-Seventh Asilomar Conference on Signals, Systems and Computers,

[11].R. Kumaresan, D.W. Tufts, Estimating the angles of arrival of multiple plane waves, IEEE Transactions on Aerospace and Electronic Systems AES-19 (1983) 134-139.

[12].C. Knapp, G. Carter, The generalized correlation method for estimation of time delay, IEEE Transaction on Acoustics, Speech, Signal Processing 24 (4) (1976) 320327

[13].A.H. Sayed, A. Tarighat, N. Khajehnouri, Network-based wireless location: challenges faced in developing techniques for accurate wireless location information, IEEE Signal Processing Magazine

[14].D. Koks, Numerical calculations for passive geolocation scenarios, Tech. Rep. DSTO-RR-0000, 2005.

[15].Do Van Giang, Tarik Taleb,Kazuo Hashimito, Nei Kato and Yoshiaki Nemoto, A Fair and Lifetime-Maximum Routing Algorithm for Wireless Sensor Network, IEEE GLOBECOM 2007 proceeding. 